\title{
EFICÁCIA DE PROCEDIMENTOS DE SUPERVISÃO PARA CONSTRUÇÃO DE TRÍPLICE CONTINGÊNCIA DE REFORÇAMENTO
}

\author{
EFFECTIVENESS OF SUPERVISING PROCEDURES IN THE DETERMINATION OF THE THREE-TERM \\ CONTINGENCIES OF REINFORCEMENT
}

\author{
TATIANA LANCE DUARTE - ORCID 0000-0003-3072-7385 \\ INSTITUTO dE TERAPIA POR CONTINGÊNCIAS DE REFORÇAMENTO - CURSO DE ESPECIALIZAÇÃO EM TERAPIA POR CONTINGÊNCIA DE \\ REFORÇAMENTO. BRASIL \\ HÉLIO JOSÉ GUILHARDI - ORCID 0000-0003-4490-9585 \\ INSTITUTO de TERAPIA POR CONTINGÊNCIAS DE REFORÇAMENTO - CURSO dE ESPECIALIZAÇÃo EM TERAPIA POR CoNTINGÊNCIA dE \\ REFORÇAMENTO. BRASIL
}

\author{
ROBERTO ALVES BANACO - ORCID 0000-0002-0619-338X \\ Paradigma Centro de CiênCias e Tecnologia do Comportamento - Mestrado Profissional em Análise do \\ COMPORTAMENTO APLICADA. BRASIL
}

\begin{abstract}
Supervisão clínica é uma atividade que promove o aprendizado do repertório de identificar, descrever, intervir e analisar as contingências de reforçamento que operam na vida do cliente. A análise de contingências de reforçamento é a ferramenta básica do analista do comportamento clínico para alcançar o objetivo de descrever as relações funcionais entre o que a pessoa faz e sente e o ambiente ao qual responde. Para identificar qual a forma mais eficaz de ensinar o terapeuta a construir a tríplice contingência de reforçamento em sessão, foram elaborados três procedimentos de supervisão. Foram definidos três grupos experimentais, sendo cada grupo composto por dois terapeutas e uma única supervisora comum aos três grupos. No Grupo 1, a supervisora usou os três procedimentos: instrução verbal, instrução verbal acrescida de autoclíticos e instrução verbal acrescida de autoclíticos e modelo; no Grupo 2, a supervisora usou dois dos procedimentos: instrução verbal acrescida de autoclíticos e instrução verbal acrescida de autoclíticos e modelo; e, no Grupo 3, a supervisora usou um procedimento: instrução verbal acrescida de autoclíticos e modelo. Os resultados encontrados sugerem que o procedimento de instrução verbal acrescida de autoclíticos e modelo foi o mais eficaz sobre o comportamento do terapeuta.
\end{abstract}

RESUMO

Palavras-chave: procedimentos de supervisão clínica; instrução verbal; autoclíticos; apresentação de modelo; terapia por contingências de reforçamento (TCR); tríplice contingência de reforçamento.

\section{ABSTRACT}

Clinical supervision is an activity that promotes the learning of the repertory of identifying, describing, analyzing and dealing with the contingencies of reinforcement that operates in the life of the client. The analysis of reinforcement contingencies is the behavior analyst's basic tool of the clinical behavior analyst to achieve the objective of describing the functional relations between what the person does and feels and the environment to which he responds. Trying to identify the most effective way to teach the therapist to build the three-term reinforcement contingency in a session, three supervising procedures were developed. Three experimental groups were defined, each group consisting of two therapists and a single supervisor common to the three groups. In Group 1 the supervisor used three procedures: verbal instruction, verbal instruction plus autoclitics, and verbal instruction plus autoclitics and model. In Group 2 the supervisor used two of the procedures: verbal instruction plus autoclitics, and verbal instruction plus autoclitics and model. In Group 3 the supervisor used the one procedure: verbal instruction plus autoclitics and model. The results found suggest that procedure of verbal instruction plus autoclitics and model was the most effective on the psychotherapist's behavior.

Keywords: clinical supervision procedures; verbal instruction, autoclitics; model presentation; therapy by contingencies of reinforcement (TCR); three-term contingency of reinforcement.

Essa pesquisa faz parte do programa científico do Mestrado Profissional em Análise do Comportamento Aplicada (PARADIGMA) em parceria com o Curso de Especialização em Terapia por Contingências de Reforçamento (ITCR), do qual o segundo autor faz parte como Diretor do Instituto de Terapia por Contingências de Reforçamento e o terceiro autor como diretor do Paradigma - Centro de Ciência e Tecnologia do Comportamento.

Correspondência para: Tatiana Lance Duarte. E-mail: tatiana_lance@yahoo.com.br

DOI: http://dx.doi.org/10.18542/rebac.v15i1.8672 
Considera-se que uma das grandes dificuldades encontradas no desenvolvimento da psicologia como ciência e profissão é conseguir garantir a competência de formação dos futuros psicólogos (Campos, 1998). No campo da psicoterapia, diversos estudos têm procurado descrever as várias habilidades de um psicoterapeuta, sugerindo uma preocupação com a sistematização do ensino da Terapia Comportamental, uma vez que esse processo sempre foi realizado de maneira empírica, mas sem uma atenção à sua operacionalização (Bitondi \& Setem, 2007).

Segundo Moreira (2003), a supervisão clínica é a etapa culminante do treino de terapeutas, sendo, portanto, considerada como indispensável na formação de psicólogos clínicos. Por isso, a atividade está se tornando muito frequente entre os psicólogos e sendo considerada fundamental para a formação do futuro profissional. Embora seja uma atividade utilizada com frequência pelos psicólogos clínicos, não existe uma padronização de procedimentos de supervisão, o que leva certamente a uma diversidade de metodologias ocasionada por diversos fatores, como referencial teórico, formação pessoal do supervisor, ambiente onde se realiza a atividade e ausência de modelos devidamente pesquisados.

Rosenberg (2006) diz que a supervisão clínica exige grande dedicação dos supervisores em psicologia para buscar métodos capazes de proporcionar aos futuros terapeutas o aprendizado de conceitos e habilidades necessárias para a condução de um processo terapêutico efetivo. Alguns comportamentos desejáveis do terapeuta são eminentemente do tipo aberto (expresso) e podem ser facilmente treinados, seja por seguimento de regras, modelagem em role-playing, ou mesmo por modelação (Banaco, 1993).

Na literatura disponível não existe um consenso, uma metodologia única ou mesmo uma eleita como mais eficiente para treinar psicoterapeutas em formação ou recém-formados. A falta de padronização de procedimentos de intervenção que possam ser usados por supervisores para modelar o repertório do terapeuta e a ausência de um modelo de supervisão sistematizado de atuação deixam lacunas significativas na prática da supervisão clínica. Beckert (2002) menciona que, embora exista um número considerável de escritos teóricos sobre o processo de supervisão, os estudos sobre o tema limitamse a descrever experiências de ensino, existindo pouca literatura, principalmente nacional, que avalie procedimentos de supervisão [nosso destaque].

Sabe-se, com a experiência prática, que a supervisão pautada no relato verbal do supervisionado parece ser o meio mais comumente utilizado para o ensino e orientação da prática clínica dos terapeutas. De forma ampla, pode-se dizer que o processo de supervisão de terapia ocorre por meio de relatos da sessão terapêutica feitos pelo supervisionado. O supervisor ouve o relato do terapeuta sobre os acontecimentos da sessão terapêutica, analisa o comportamento do terapeuta e dá as orientações devidas (Moreira, 2003). Ou seja, também o supervisor se limita a dar orientações verbais para o supervisionado.
Alguns autores, como Barker, Pistrang e Elliot (1994) e De Rose (1997), apontam limites nos estudos baseados em relatos verbais, principalmente com relação à veracidade dos relatos. Mas, ainda assim, podemos considerar ser o comportamento verbal um componente essencial dentro da interação supervisor e supervisionado.

O contexto clínico parece ser um campo fértil para o estudo do episódio verbal estabelecido entre falante e ouvinte, revezando-se entre terapeuta e cliente, bem como no contexto entre supervisor e supervisionado, pois, neles, duas ou mais pessoas se comportam e esses comportamentos são predominantemente verbais. Tornase, assim, imprescindível analisar as relações de controle existentes entre o comportamento verbal do cliente e o comportamento verbal do terapeuta, ou do supervisor e do supervisionado. E, para tal, a supervisão pode ser um instrumento fundamental para auxiliar e direcionar o terapeuta, bem como o cliente, na compreensão das relações existentes dentro da terapia.

O método de supervisão a ser adotado e investigado no presente estudo será o baseado na Terapia por Contingência de Reforçamento (TCR), desenvolvida por Guilhardi (2004). A proposta básica da TCR é que o terapeuta comportamental, embora se interesse pelos comportamentos e sentimentos do cliente, não trabalha diretamente com eles. Tem que fazê-lo lidando com as contingências de reforçamento das quais comportamentos e sentimentos são função. $O$ instrumento de ação de que o terapeuta dispõe no processo terapêutico são as contingências de reforçamento (Matos, 1997). E as contingências de reforçamento são compreendidas como unidades básicas de análise e compreensão dos comportamentos e sentimentos humanos. O comportamento não está na ação em si, mas naquilo que o determina. Sendo assim, para compreender uma ação e alçá-la ao status de comportamento, temos que relacionála com eventos antecedentes e eventos consequentes a ela funcionalmente interligados. A contingência de reforçamento simples é composta por três termos, daí o nome de tríplice contingência de reforçamento. $\mathrm{O}$ primeiro termo é o antecedente, o segundo é a resposta e o terceiro é a consequência. Quando os três termos são conhecidos, e são determinadas as interações entre eles, pode-se dizer que comportamentos e sentimentos, por eles determinados, foram "explicados" (Guilhardi, 2004).

A análise funcional é o instrumento básico de trabalho do analista do comportamento que atua na clínica, identificando e manejando contingências em operação na vida do cliente (Meyer, 2009). A busca das variáveis externas (ambientais, chamadas, também, de independentes), das quais o comportamento (variável dependente) é função, consiste na principal finalidade de uma análise funcional (Skinner, 1953/2003). Sendo assim, uma das vantagens de uma análise funcional é a identificação de variáveis que influenciam na ocorrência do comportamento de interesse, tornando possível o planejamento de intervenções, a modificação dessas variáveis e do comportamento-problema, e a previsão das condições que podem proporcionar a generalização e a manutenção das modificações comportamentais efetuadas. 
Nessa perspectiva, em coerência com os marcos conceituais de Skinner, o objetivo da Análise do Comportamento é identificar e trabalhar com as contingências de reforçamento, sendo que estas não se restringem a contextos experimentais. A partir da análise de contingências de reforçamento, podem-se inferir as contingências que operaram na história de vida do cliente e estabelecer novas relações de contingências, visando produzir mudanças e instalar novos comportamentos desejáveis.

Cabe enfatizar que o objeto de estudo e de interesse do terapeuta é o comportamento humano; e o instrumento de análise usada pelo terapeuta analista do comportamento para atuar na clínica é a análise de contingências, que se caracteriza pela descrição minuciosa dos termos que compõem a tríplice contingência de reforçamento e no relato das interações funcionais entre seus termos (Guilhardi, 2004).

Os procedimentos de supervisão clínica selecionados para compor o presente estudo (descritos a seguir) constituem na adoção de procedimentos consolidados na literatura como sendo eficientes para instalação de repertórios, fundamentados pelos princípios que regem os comportamentos humanos.

Segundo Catania (1999), a mais ampla função da linguagem é a instrução. Instrução verbal consiste na apresentação, pelo falante, de verbalizações e descrições sobre contingências de reforçamento, mais especificamente sobre respostas a serem emitidas pelo ouvinte em determinadas condições antecedentes. Tais verbalizações têm a função de evento antecedente para o ouvinte que, sob controle delas, emitirá os comportamentos especificados nas instruções. Ao comportar-se sob controle das instruções verbais, o ouvinte (aprendiz) pode ter seu comportamento modificado sem ter o contato direto com as consequências naturais de seus comportamentos. As instruções (antecedentes verbais) passam a exercer controle sobre o comportamento do ouvinte, e as contingências operam sobre o comportamento de seguir as instruções (Catania, 1999).

$\mathrm{O}$ operante verbal autoclítico (Skinner (1957/1978) pode funcionar como instruções ao ouvinte, que o ajudam a se comportar de uma maneira que torna mais provável a obtenção de consequências reforçadoras. Os autoclíticos produzem efeito sobre o comportamento do ouvinte. Dois seis subtipos de classes verbais de autoclíticos, o subtipo descritivo é relevante para o presente estudo. Segundo Borloti (2004), os autoclíticos descritivos são discriminações de comportamentos do falante; eles informam o ouvinte acerca do tipo de operante verbal emitido pelo falante (Skinner, 1957/1978).

Outro procedimento eficaz na instalação de novos repertórios é a apresentação de modelos. Quando um indivíduo emite o comportamento que foi apresentado por um outro indivíduo, seu comportamento compõe a classe de imitação. Quando esta imitação não se restringe às condições que correspondem à situação inicial em que o modelo foi apresentado, o comportamento de seguir o modelo passa a compor a classe de imitação generalizada, uma classe de comportamentos de ordem superior (Catania, 1999).

As vantagens do uso de procedimentos de instrução e de apresentação de modelos repousam sobre o fato de que o aprendizado de novos comportamentos pode ser otimizado, ocorrendo de forma rápida, eficiente (no caso da apresentação de modelos) e sem contato direto com as contingências naturais (no caso das instruções verbais) (Catania, 1999).

Nessa direção, este estudo teve como objetivo utilizar três procedimentos de supervisão clínica com os participantes (terapeutas iniciantes ou recém-formados) e investigar qual destes procedimentos (P1, P2 e P3, descritos a seguir) foi mais eficaz para ensinar os participantes o comportamento desejado. Os procedimentos utilizados pela supervisora com os terapeutas em supervisão clínica descritos neste trabalho consistiram em: apresentação de instruções (P1), apresentação de instruções acrescida do uso de autoclíticos (P2), e apresentação de instruções acrescida do uso de autoclíticos, somada à apresentação de modelos (P3). O comportamento final esperado dos terapeutas era construir e apresentar a tríplice contingência de reforçamento de uma das dificuldades comportamentais relatadas pelo cliente em sessão.

Cabe ressaltar que não foram encontrados estudos publicados relacionados aos objetivos da presente investigação.

\section{Considerações éticas}

\section{MÉTODO}

Tanto os terapeutas como as clientes foram recrutados em contexto de aula, em que lhes foi perguntado: "Quem gostaria de participar voluntariamente de uma pesquisa em clínica, atuando como terapeuta ou 'cliente' (no segundo caso, com desempenho simulado), durante o primeiro semestre de 2017 numa atividade que exigirá duas horas de dedicação semanal (em média) durante três meses?". A exigência de que as "clientes" apresentassem queixas simuladas foi feita para não as expor com queixas reais minimizando, dessa forma, problemas éticos.

Todos os participantes que aceitaram as condições desta pesquisa, declararam estar de acordo mediante a assinatura do Termo de Consentimento Livre e Esclarecido. Nesta pesquisa não houve procedimentos correlacionados ao processo real de terapia do cliente, visto que o processo utilizado como material base para as análises se tratou de informações coletadas com queixas simuladas.

Inicialmente, o projeto foi submetido à aprovação da Banca de Qualificação constituída na Instituição e Associação Paradigma - Centro de Ciência e Tecnologia do Comportamento e, posteriormente, à aprovação do Comitê de Ética da Plataforma Brasil (CAEE 68817317.5.000.5374). Após receber parecer favorável desses órgãos, a pesquisadora iniciou a etapa de seleção dos participantes. 


\section{Participantes}

Participaram deste estudo a supervisora, seis alunos-terapeutas, duas clientes, três juízes e uma auxiliar com formação em Psicologia para fazer as transcrições das supervisões. A pesquisadora deste estudo foi a própria supervisora, formada há 15 anos, Especialista em Terapia por Contingências de Reforçamento e que vem atuando, desde então, como psicóloga clínica no Instituto de Terapia por Contingências de Reforçamento e como supervisora dos cursos de Formação e Especialização no mesmo Instituto. Os seis terapeutas voluntários (ambos os sexos) estavam, obrigatoriamente, matriculados no curso de Formação ou Especialização do ITCR - Campinas e cursando graduação em Psicologia (um participante cursava o $4^{\circ}$ ano e dois participantes cursavam o $5^{\circ}$ ano) ou na condição de recémformado (até dois anos de formação) (três participantes). As duas clientes voluntárias (ambas do sexo feminino) estavam matriculadas no curso de Especialização do ITCR Campinas e atuaram como se fossem "atrizes" com uma queixa simulada. Os três juízes avaliadores que participaram deste estudo tinham mais de três anos de experiência como supervisores e psicólogos clínicos, sendo também formados em Terapia por Contingências de Reforçamento com atuação no Instituto de Terapia por Contingências de Reforçamento de Campinas.

\section{Critérios de inclusão dos participantes}

Os terapeutas selecionados para a pesquisa atendiam ao critério de nunca terem exercido a função de terapeuta, além das experiências nos estágios clínicos exigidos no curso de graduação. Todos atendiam aos critérios quanto ao tempo de formação: estar nos dois últimos anos do curso de graduação em Psicologia ou ter se formado em no máximo dois anos.

\section{Coleta de dados}

A coleta de dados foi feita a partir das gravações (vídeo e áudio) e transcrições das supervisões e das sessões de atendimento. As transcrições das supervisões foram feitas pela auxiliar (psicóloga) treinada especialmente para essa função, e as transcrições dos atendimentos foram feitas pelos próprios terapeutas. A supervisora, aleatoriamente, comparou trechos curtos entre todas as transcrições e vídeos das supervisões e de atendimentos a fim de ter uma amostra da fidedignidade dos registros. Observou-se que todos os trechos selecionados estavam transcritos de forma fidedigna ao vídeo correspondente.

\section{Procedimento geral}

Foram então constituídas as seis tríades com Supervisora - Terapeuta - Cliente, distribuídas em três grupos (Grupo 1, Grupo 2 e Grupo 3). Cada um dos grupos foi constituído da seguinte maneira:

Grupo 1 - Supervisora - Terapeuta 1 - Cliente 1 Supervisora - Terapeuta 2 - Cliente 2

Grupo 2 - Supervisora - Terapeuta 3 - Cliente 1 Supervisora - Terapeuta 4 - Cliente 2

Grupo 3 - Supervisora - Terapeuta 5 - Cliente 1 Supervisora - Terapeuta 6 - Cliente 2
Em seguida, os terapeutas foram individualmente convocados por e-mail para assistirem a uma aula teórica gravada em DVD, contendo Conceitos Básicos da Análise do Comportamento relacionados aos objetivos da pesquisa: queixa espontânea da cliente, dificuldade comportamental, episódio comportamental, os três termos da tríplice contingência e a inter-relação entre eles. Esperava-se, com essa aula, um nivelamento conceitual básico de todos os terapeutas envolvidos nesta pesquisa antes de iniciar a fase de aplicação dos procedimentos. Os terapeutas poderiam rever o vídeo quantas vezes fossem necessárias. Quando o terapeuta se declarou apto para se submeter a uma avaliação, foi lhe pedido que respondesse individualmente, por escrito, seis questões dissertativas com o objetivo de verificar o aprendizado dos conceitos ensinados na aula gravada. $\mathrm{O}$ critério de acerto às perguntas da avaliação era de $100 \%$. O participante poderia repetir a avaliação até atingir o critério de desempenho estipulado.

Após essa fase, cada terapeuta recebeu um $e$-mail contendo orientações sobre dia, horário e sala onde ocorreriam as atividades. Além disso, o e-mail continha um anexo com o primeiro nome da "cliente" que iria atender e uma síntese por escrito, elaborada pela supervisora, das duas queixas que a "cliente" iria expor para o terapeuta na sessão. Outro anexo contido neste $e$ mail foi o roteiro que descrevia os procedimentos a serem empregados na sessão. Na supervisão, que antecedia o atendimento propriamente dito, a supervisora lia e discutia com o terapeuta todo o material escrito. Esse $e$-mail foi enviado com, no mínimo, $24 \mathrm{~h}$ de antecedência, com o objetivo de permitir que os terapeutas pudessem ler, estudar e se preparar para a supervisão e para a sessão de terapia.

A pesquisa envolveu as seguintes fases: (a) Atendimento Livre: realização da primeira supervisão, individualmente, na qual o participante (terapeuta) recebia da supervisora instruções básicas para o primeiro atendimento (apresentar-se à cliente, ouvir as queixas etc.); (b) Procedimento 1: realização de supervisão, individualmente, na qual o participante recebia da supervisora tão somente instruções (orais e textuais) para conduzir o atendimento; (c) Procedimento 2: realização de supervisão, individualmente, na qual o participante recebia da supervisora as mesmas instruções (orais e textuais) do Procedimento 1, acrescidas de autoclíticos (os autoclíticos utilizados foram as palavras escritas em negrito e sublinhadas), para o atendimento; (d) Procedimento 3: realização de supervisão, individualmente, na qual o participante recebia da supervisora as mesmas instruções (orais e textuais) dadas nos Procedimentos 1 e 2, acrescidas de autoclíticos (os autoclíticos utilizados foram as palavras escritas em negrito e sublinhadas) da mesma forma que no Procedimento 2, além da apresentação de modelos (os modelos fornecidos de como executar a instrução eram fornecido pela supervisora considerando para tal um caso e de queixas fictícios) para conduzir o atendimento.

Abaixo, segue como exemplo, a mesma instrução, e neste caso selecionamos como exemplo a instrução 2, 
fornecida tanto no Procedimento 1, quanto no Procedimento 2 e no Procedimento 3:

Procedimento 1. Instrução 2: Em seguida, repita para sua cliente, com suas próprias palavras, as duas queixas que ela relatou na primeira sessão.

Procedimento 2. Instrução 2: EM SEGUIDA, REPITA para sua cliente, COM SUAS PRÓPRIAS PALAVRAS as DUAS QUEIXAS que ela relatou na primeira sessão.

Procedimento 3. Instrução 2: No início da sessão, VERBALIZE para sua cliente AS QUEIXAS que ela relatou na primeira sessão. MODELO: DIGA, POR EXEMPLO: "FLÁVIA, NA ÚLTIMA SESSÃO VOCÊ ME APRESENTOU DUAS QUEIXAS. UMA, FOI SUA TRISTEZA SEM MOTIVO APARENTE E A OUTRA, FOI SUA DIFICULDADE NA RELAÇÃO COM SEU CHEFE." AGORA FAÇA IGUAL A MIM, com os dados de sua cliente.
O atendimento livre foi realizado em uma sessão, e cada tipo de supervisão foi seguido por uma sessão de atendimento ao cliente. Desta forma, os terapeutas do Grupo 1 realizaram o total de 4 sessões de atendimento; os terapeutas do Grupo 2, 3 sessões, e os terapeutas do Grupo 3 realizaram 2 sessões de atendimento.

As sessões realizadas pelos terapeutas eram gravadas em áudio e vídeo. A pesquisadora (supervisora) lia o conteúdo das transcrições das sessões e assistia aos vídeos antes da supervisão seguinte. O único tipo de consequência apresentada pela supervisora era a verbalização: "Li sua transcrição e assisti ao vídeo da sessão. Os objetivos de sua sessão foram cumpridos. Podemos seguir adiante!".

A Tabela 1 contém a disposição dos seis participantes nos três grupos que compuseram o estudo, de acordo com o tipo de procedimento a que cada um deles foi submetido.

Tabela 1.

Distribuição dos participantes nos grupos de acordo com os procedimentos realizados.

\begin{tabular}{cccccc}
\hline Grupo & Participante & $\begin{array}{c}\text { Atendimento } \\
\text { Livre }\end{array}$ & $\begin{array}{c}\text { Procedimento } \\
\text { 1 (instrução) }\end{array}$ & $\begin{array}{c}\text { Procedimento 2 } \\
\text { (instrução }+ \\
\text { autoclítico) }\end{array}$ & $\begin{array}{c}\text { Procedimento 3 } \\
\text { (instrução }+ \\
\text { autoclítico + } \\
\text { modelo) }\end{array}$ \\
\hline 1 & 1 & $\mathrm{X}$ & $\mathrm{X}$ & $\mathrm{X}$ & $\mathrm{X}$ \\
& 2 & $\mathrm{X}$ & $\mathrm{X}$ & $\mathrm{X}$ & $\mathrm{X}$ \\
2 & 3 & $\mathrm{X}$ & $\mathrm{X}$ & $\mathrm{X}$ \\
& 4 & $\mathrm{X}$ & $\mathrm{X}$ & $\mathrm{X}$ \\
3 & 5 & $\mathrm{X}$ & & & $\mathrm{X}$ \\
\hline
\end{tabular}

Os procedimentos utilizados com todos os participantes (Grupos 1, 2 e 3) tiveram como objetivo instalar comportamentos no terapeuta de construir por escrito e apresentar oralmente para a cliente a tríplice contingência de reforçamento de um episódio comportamental selecionado em sessão. Os objetivos do presente estudo consistiram em comparar a eficácia das diferentes condições antecedentes (Procedimentos 1, 2 e 3 ) para a instalação dos comportamentos-alvo nos terapeutas participantes.

\section{Procedimentos de registro e análise de dados}

Os dados foram observados, registrados e analisados para cada um dos Grupos (1, 2 e 3) em duas categorias: a) comportamentos da supervisora conforme categorização descrita; e b) comportamentos do terapeuta conforme categorização descrita.

Os dados foram coletados comparando-se a ocorrência ou não ocorrência dos comportamentos sob estudo emitidos pela supervisora e pelos terapeutas nos atendimentos livres e nos procedimentos P1, P2 e P3. Os registros foram feitos pelos juízes em duas folhas de registro distintas: uma para a supervisora e outra para os terapeutas.

Os dados, assim coletados, deveriam indicar: a) se a supervisora se comportou nas supervisões de forma consistente com as instruções referentes a cada fase do procedimento; e b) se houve ou não diferenças nos comportamentos-alvo dos terapeutas em função dos diferentes procedimentos experimentais aplicados.

Os comportamentos dos terapeutas, cuja ocorrência deveria ser registrada pelos juízes, foram classificados em duas categorias: comportamentos gerais, isto é, aqueles que são esperados na interação psicoterapeuta-cliente, mas que não têm relação essencial com o objetivo final do procedimento (construir e apresentar a tríplice contingência de reforçamento); e comportamentos específicos, isto é, comportamentos que compõem o objetivo comportamental do procedimento (variável dependente).

$\mathrm{O}$ estudo empregou um delineamento entregrupos. Nesse delineamento, os efeitos de uma condição experimental são avaliados pela comparação entre diferentes grupos de sujeitos, submetidos, cada um deles, a diferentes condições. Nesse delineamento, cada sujeito foi exposto a apenas uma das condições do experimento e, além disso, os dois sujeitos do Grupo 1, os dois sujeitos do Grupo 2 e os dois sujeitos do Grupo 3 foram expostos às mesmas condições por um mesmo período de tempo. $\mathrm{O}$ delineamento experimental entre-grupos permitiu comparar os efeitos da introdução das variáveis experimentais. De acordo com a Tabela 1, também é 
possível observar que o Grupo 1 foi introduzido a cada variável experimental sucessivamente; o Grupo 2 passou pela introdução simultânea de duas das três variáveis experimentais do Grupo 1 em sucessão; o Grupo 3 passou pela introdução simultânea das três variáveis experimentais do Grupo 1.

\section{RESULTADOS}

Os resultados são apresentados na forma de tabelas. Na Tabela 2 são apresentados o número total de comportamentos avaliados e a porcentagem de comportamentos emitidos por cada terapeuta do Grupo 1 na etapa de Atendimento Livre (AL) e nos três procedimentos experimentais.

De acordo com a Tabela 2, o Procedimento 1 não foi suficiente para o terapeuta construir por escrito e apresentar oralmente a tríplice contingência de reforçamento, uma vez que o Terapeuta 1 emitiu apenas $38 \%$ dos comportamentos específicos esperados, e o Terapeuta 2, apenas $46 \%$. Após passar pelo Procedimento 2, os Terapeutas 1 e 2 apresentaram mais comportamentos específicos desejados $(69 \%$ e $76 \%$, respectivamente). Porém, tal aumento não foi suficiente para o terapeuta construir por escrito e apresentar oralmente a tríplice contingência de reforçamento. Após a realização do Procedimento 3 , foi possível observar uma melhora no desempenho de ambos os terapeutas em relação ao uso exclusivo do Procedimento 2 na emissão dos comportamentos do objetivo do procedimento. O Terapeuta 2 construiu por escrito e apresentou oralmente a tríplice contingência de reforçamento, apresentando $93 \%$ dos comportamentosalvo esperados.

Tabela 2.

Comportamentos emitidos pelos dois terapeutas do Grupo 1 no atendimento livre e nas diferentes fases dos procedimentos experimentais.

\begin{tabular}{ccccccccccc}
\hline & $\begin{array}{c}\text { Unidade } \\
\text { de } \\
\text { Medida }\end{array}$ & \multicolumn{3}{c}{ Terapeuta 1 } & \multicolumn{5}{c}{ Terapeuta 2 } \\
\hline $\begin{array}{c}\text { Etapa do } \\
\text { procedimento }\end{array}$ & & AL & P1 & P2 & P3 & AL & P1 & P2 & P3 \\
Comportamentos & $\%$ & 100 & 100 & 100 & 100 & 100 & 100 & 100 & 100 \\
Gerais & $\mathrm{N}$ & 6 & 5 & 5 & 7 & 6 & 5 & 5 & 7 \\
Comportamentos & $\%$ & -- & 38 & 69 & 70 & -- & 46 & 76 & 93 \\
Específicos & $\mathrm{N}$ & -- & 13 & 13 & 14 & -- & 13 & 13 & 14 \\
\hline
\end{tabular}

Nota: AL: Atendimento livre; \%: Percentual de comportamentos emitidos; N: Total de comportamentos avaliados.

De acordo com a Tabela 3, é possível observar que o Procedimento 2 realizado com o Grupo 2 não foi suficiente para os terapeutas construírem por escrito e apresentarem oralmente a tríplice contingência de reforçamento (o Terapeuta 3 apresentou $23 \%$ dos comportamentos-alvo, e o Terapeuta 4, 54\%). O Procedimento 3 produziu uma melhora em ambos os terapeutas em relação ao uso exclusivo do Procedimento 2 na emissão dos comportamentos do objetivo do procedimento (o Terapeuta 3 passou a apresentar $79 \%$ dos comportamentos-alvo, e o Terapeuta 4, 93\%). O Terapeuta 4 conseguiu atingir o desempenho terapêutico funcional, ou seja, o terapeuta construiu por escrito e apresentou oralmente a tríplice contingência de reforçamento.

Tabela 3.

Comportamentos emitidos pelos dois terapeutas do Grupo 2 no atendimento livre e nas diferentes fases dos procedimentos experimentais.

\begin{tabular}{cccccccccc}
\hline & $\begin{array}{c}\text { Unidade } \\
\text { de } \\
\text { Medida }\end{array}$ & & & Terapeuta 3 & \multicolumn{5}{c}{ Terapeuta 4} \\
\hline $\begin{array}{c}\text { Etapa do } \\
\text { procedimento }\end{array}$ & & AL & P1 & P2 & P3 & AL & P1 & P2 & P3 \\
Comportamentos & $\%$ & 85 & -- & 100 & 100 & 83 & -- & 100 & 100 \\
Gerais & N & 6 & -- & 5 & 7 & 6 & -- & 5 & 7 \\
Comportamentos & $\%$ & -- & -- & 23 & 79 & -- & -- & 54 & 93 \\
Específicos & $\mathrm{N}$ & -- & -- & 13 & 14 & -- & -- & 13 & 14 \\
\hline
\end{tabular}

Nota: AL: Atendimento livre; \%: Percentual de comportamentos emitidos; N: Total de comportamentos avaliados.

O Procedimento 2, introduzido sem a experiência prévia do Procedimento 1, produziu para os dois terapeutas do Grupo 2 uma porcentagem menor na emissão dos comportamentos-alvo do procedimento do que quando houve uma sessão de atendimento anterior feita com instrução (Grupo 1): após a realização do Procedimento 2, os Terapeutas 3 e 4 (Grupo 2) apresentaram $23 \%$ e $54 \%$ de desempenho, respectivamente, enquanto os Terapeutas 1 e 2 (Grupo 1) apresentaram $69 \%$ e $76 \%$ dos comportamentos-alvo, 
respectivamente. Estes dados sugerem que a introdução em sequência das variáveis instrução e instrução mais autoclítico pode ser mais eficiente do que a introdução direta dos dois procedimentos associados.

A introdução do Procedimento 3, quer tenha sido precedida dos procedimentos 1 e 2 (Grupo 1), quer tenha sido precedida apenas do Procedimento 2 (Grupo 2), produziu idênticos resultados (Terapeuta 1 apresentou
$70 \%$ dos comportamentos avaliados, Terapeuta 2, 93\%; no Grupo 2, o Terapeuta 3 apresentou $79 \%$ dos comportamentos avaliados, e o Terapeuta 4, 93\%). Isso sugere que a introdução isolada do Procedimento 1 não alterou o desempenho no Procedimento 3 , ou seja, pode ser dispensada, assim como o Procedimento 2 pode ser dispensado, pois ele mesmo, isoladamente, não atingiu o objetivo.

Tabela 4.

Comportamentos emitidos pelos dois terapeutas do Grupo 3 no atendimento livre e nas diferentes fases dos procedimentos experimentais.

\begin{tabular}{cccccccccc}
\hline & $\begin{array}{c}\text { Unidade } \\
\text { de } \\
\text { Medida }\end{array}$ & \multicolumn{3}{c}{ Terapeuta 5 } & & \multicolumn{3}{c}{ Terapeuta 6 } \\
\hline $\begin{array}{c}\text { Etapa do } \\
\text { procedimento }\end{array}$ & & AL & P1 & P2 & P3 & AL & P1 & P2 & P3 \\
Comportamentos & $\%$ & 100 & -- & -- & 100 & 83 & -- & -- & 86 \\
Gerais & $\mathrm{N}$ & 6 & -- & -- & 7 & 6 & -- & -- & 7 \\
Comportamentos & $\%$ & -- & -- & -- & 93 & -- & -- & -- & 93 \\
Específicos & $\mathrm{N}$ & -- & -- & -- & 14 & -- & -- & -- & 14 \\
\hline
\end{tabular}

Nota: AL: Atendimento livre; \%: Percentual de comportamentos emitidos; N: Total de comportamentos avaliados.

De acordo com a Tabela 4, o Procedimento 3, que incluiu todos os procedimentos numa única supervisão de treinamento e que foi conduzido em uma única sessão com o cliente, apresentou, para os dois terapeutas, resultados terapêuticos funcionais, ou seja, ambos conseguiram construir por escrito e apresentar oralmente a tríplice contingência de reforçamento. Os Terapeutas 5 e 6 apresentaram $93 \%$ dos comportamentos específicos ensinados pelos procedimentos e avaliados.

Para apresentar os resultados referentes aos dados de qual procedimento teve maior eficácia sobre o comportamento do terapeuta, foram realizadas análises das respostas emitidas pelo comportamento da supervisora em supervisão que evocaram as respostas emitidas pelo comportamento dos terapeutas em sessões de terapia. Sendo assim, os terapeutas 2, 4, 5 e 6 , participantes da pesquisa, atingiram o objetivo de construir por escrito e apresentar oralmente a tríplice contingência de reforçamento, conforme exigido pelo estudo. Todos os quatro terapeutas foram submetidos aos três procedimentos: instrução, instrução mais autoclítico, instrução mais autoclítico e modelo. O Procedimento 1 exigiu três sessões de atendimento para que o Terapeuta 2 chegasse ao desempenho final esperado; o Procedimento 2 exigiu duas sessões para que o Terapeuta 4 chegasse ao desempenho final esperado; o Procedimento 3 exigiu apenas uma sessão para que os Terapeutas 5 e 6 chegassem ao desempenho final esperado. A introdução do Procedimento 3, quer tenha sido precedida dos procedimentos 1 e 2 (Grupo 1), quer tenha sido precedida apenas do Procedimento 2, produziu idênticos resultados. Isso sugere que a introdução isolada do Procedimento 1 não alterou o desempenho no Procedimento 3, ou seja, pode ser dispensada.

Os resultados, portanto, indicam que: 1) Supervisão apenas com uso de instrução não basta; 2) Supervisão apenas com uso de instrução mais autoclítico não basta; 3) Supervisão com instrução mais autoclítico, seguida de uma primeira sessão de atendimento, acrescida de uma segunda supervisão com uso de instrução mais autoclítico e modelo seguida de uma segunda sessão de atendimento pode bastar; 4) Supervisão com uso de instrução mais autoclítico mais modelos seguida de uma única sessão de atendimento basta.

Essas conclusões se baseiam em avaliações feitas com uma amostra de dois terapeutas em cada condição. Deve-se ter cuidado com qualquer generalização desses dados como válidos para uma amostra mais abrangente. As diferenças de resultados podem se dever a diferenças na maneira de introduzir os procedimentos, a diferenças de repertórios comportamentais terapêuticos não identificadas entre os sujeitos e a outras possíveis variáveis não identificadas.

Convém destacar que os Terapeutas 2 e 6 , que construíram a tríplice contingência de reforçamento, cursavam o $5^{\circ}$ ano da graduação; o Terapeuta 4, que construiu a tríplice contingência de reforçamento, cursava o $4^{\circ}$ ano; e o Terapeuta 5 , que construiu a tríplice contingência de reforçamento, era recém-formado. Os Terapeutas 2, 4 e 6 estavam frequentando pela primeira vez o curso de formação de terapeutas comportamentais, e o Terapeuta 5 estava frequentando o curso de especialização. Os Terapeutas 1 e 3 , que não conseguiram construir a tríplice de contingência de reforçamento, tinham iniciado o curso de formação; o Terapeuta 1 frequentava o $4^{\circ}$ ano de graduação e o Terapeuta 3 era recém-formado.

Recomenda-se que os supervisores adotem três procedimentos em conjunto, a fim de conseguir o desempenho comportamental final desejado com menos sessões de supervisão e sugere-se a replicação dos procedimentos com um maior número de participantes, em particular do Procedimento 3. 


\section{DISCUSSÃO}

Os resultados apresentados neste trabalho contribuem para ampliar a compreensão do papel fundamental da escolha de procedimentos específicos usados pelo supervisor em supervisão que irão ensinar ou não o terapeuta a construir por escrito e apresentar oralmente para o cliente a tríplice contingência de reforçamento do episódio comportamental selecionado.

Foram utilizados procedimentos experimentais tecnologicamente descritos e conceitualmente sistemáticos (Baer, Wolf \& Risley, 1968), o que os torna replicáveis e consistentes com a Análise do Comportamento. Todos os procedimentos foram descritos tecnologicamente, não apenas instrução verbal do comportamento. Os três procedimentos experimentais (instrução verbal, instrução verbal com uso de autoclíticos e instrução verbal com uso de autoclíticos e acompanhada de modelos) foram apresentados em instruções textuais (e adicionalmente orais) para os terapeutas. Tendo isso em vista, pode-se considerar que o presente estudo contribuiu para produzir informações acerca dos procedimentos de supervisão, apontadas por Beckert (2002) como sendo escassas na literatura nacional.

As instruções orais e textuais utilizadas pela supervisora nos procedimentos tiveram como objetivo fornecer aos terapeutas orientações específicas de como se comportar nas sessões, evitando, desta forma, que se mantivessem apenas sob controle de contingências naturais aleatórias que caracterizam uma interação desvinculada de um sólido referencial conceitual e de evidências experimentais. Como escreveu Skinner (1957/1978, pp. 436-437): “o ouvinte é instruído pela repetição, pelas técnicas de instigação e investigação".

Também se investigaram formas mais elaboradas de instrução verbal enriquecidas com autoclíticos. A escolha e o uso de autoclíticos como um outro procedimento específico, que visa a enfatizar e facilitar a compreensão das instruções aos psicoterapeutas, deram-se para investigar se essas variáveis bastariam para controlar o comportamento do supervisionando dentro da sessão. Como mencionou Skinner (1957/1978 p.411): “A explicação definitiva do comportamento autoclítico repousa no efeito que ele exerce sobre o ouvinte". A emissão de comportamentos verbais autoclíticos pela supervisora mostrou ser uma variável importante para evocar os comportamentos desejados do terapeuta em sessão.

Por fim, a escolha do uso de modelos fornecidos pela supervisora objetivou dar um passo além, qual seja, apresentar modelos dados pela supervisora. Ao apresentar um modelo, o supervisor aumenta o grau de controle sobre o supervisionado (controle pelo antecedente) e evoca o comportamento imitativo, que, emitido na presença do supervisor, permite a ele reforçar diferencialmente a emissão do comportamento até a correta imitação (seleção pelas consequências). A apresentação dos modelos tinha por objetivo mostrar para o psicoterapeuta o que era minimamente correto e essencial para ser feito. Embora, nesta pesquisa, tenha sido esperado que o terapeuta atuasse na sessão sob controle do modelo apresentado, não se pretende, a longo prazo, com o uso do procedimento de imitação, limitar a criatividade do psicoterapeuta, mas tão somente fornecerlhe um primeiro passo apropriado a ser emitido e que será progressivamente modificado e selecionado pelas consequências que produz. A eficácia da apresentação de modelos como uma das variáveis independentes do estudo corrobora as colocações de Banaco (1993) sobre a possibilidade de serem treinados comportamentos do tipo aberto (expresso) relevantes na atuação do terapeuta.

Os resultados do presente estudo revelaram que o Procedimento 3 (combinação de uso de instruções orais e textuais, acrescidas de autoclíticos e modelos) teve maior eficácia sobre o comportamento do terapeuta para construir por escrito e apresentar oralmente em sessão a tríplice contingência de reforçamento de um episódio comportamental selecionado quando comparado aos Procedimentos 1 e 2 .

A confiança na força do Procedimento 3 foi confirmada no Grupo1: quando se introduziu o Procedimento 3, um dos sujeitos replicou os dados do Grupo 3, e no Grupo 2, quando se introduziu o Procedimento 3, um dos sujeitos replicou os dados do Grupo 3.

Pode-se afirmar que, todos os procedimentos apresentados foram descritos tecnologicamente, o que permite replicações experimentais tanto diretas quanto sistemáticas (Sidman, 1960), o que é uma contribuição fértil para o desenvolvimento da área de pesquisa sobre eficácia diferenciada de diferentes procedimentos de supervisão. O estudo foi conceitualmente sistemático, como uma maneira de investigar o fenômeno comportamental de interesse pela adoção da Análise do Comportamento. O que norteou a elaboração da presente pesquisa e aplicação dos procedimentos foi um comprometimento ao lidar o tempo todo com contingências de reforçamento.

Procurou-se, no presente estudo, descrever os procedimentos com terminologia e conceitos operantes. Os autores pesquisaram o papel do controle de estímulos antecedentes sobre o comportamento dos terapeutas nos atendimentos. Para tal, utilizou regras verbais (instruções verbais), em seguida regras com autoclíticos e, depois, empregou regras com autoclíticos mais modelos. Cada uma dessas variáveis experimentais foi introduzida de forma sistemática de acordo com os procedimentos.

Nesse sentido, a ênfase da pesquisa esteve no manejo dos três diferentes componentes do primeiro termo (antecedente) da tríplice contingência. Parece que este delineamento de manejo de variáveis é o mais apropriado para o contexto em que foi realizada a pesquisa, uma vez que a supervisora não acompanhou diretamente o desempenho do terapeuta na sessão, o que impediu o uso de consequências reforçadoras diferenciais. Os diferentes procedimentos utilizados e as diferentes variáveis dependentes inseridas como condições antecedentes para os comportamentos dos terapeutas confirmam o posicionamento de Rosenberg (2006), a respeito da exigência de dedicação do 
supervisor para programar métodos eficazes de proporcionar aprendizado aos terapeutas em formação.

Nesta pesquisa, optou-se por adotar um procedimento mais corriqueiro e mais viável no treinamento de alunos nos estágios clínicos, em particular em cursos de graduação. A coleta de dados se baseou em um produto das contingências de reforçamento, no caso a construção e apresentação oral da tríplice contingência de reforçamento (objetivo final do procedimento), que, tendo sido gravada, permitiu o acesso direto aos comportamentos do terapeuta.

Os progressos no presente estudo, demonstrados pelos terapeutas que construíram a tríplice contingência de reforçamento, pode ser uma evidência de como certas contingências específicas devem ser adequadamente planejadas para um ensino mais eficaz.

Como na literatura pertinente ao tema supervisão clínica não existe uma definição de nenhum modelo sistematizado de como o supervisor deve proceder em supervisão, esta pesquisa sugere, com os resultados do Procedimento 3 , que este pode ser um primeiro modelo com rigor tecnológico, com comprometimento conceitual e funcional a ser seguido por supervisores nas atividades de supervisão para instalar comportamentos terapêuticos apropriados aos atendimentos que realizam dentro do modelo de Terapia Comportamental.

Cabe esclarecer que o Procedimento 3 (uso de instrução acrescida de autoclíticos e modelo) que foi adotado neste experimento, envolveu a apresentação única e exclusiva de um antecedente, que foi um modelo acrescido de instruções com autoclíticos sem apresentação de uma consequência diferenciada, quer seja na sessão ou na supervisão. A única consequência apresentada no início da supervisão após a ocorrência da sessão foi: " $L i$ sua transcrição e assisti ao vídeo da sessão. Os objetivos de sua sessão foram cumpridos. Podemos seguir adiante!".

Diante de tal esclarecimento, o Procedimento 3 utilizado se aproxima do conceito de imitação generalizada. A imitação generalizada foi estudada inicialmente por Baer \& Sherman (1964), na qual esses autores demonstraram com crianças que se consegue instalar um comportamento imitativo através do manejo da tríplice contingência de reforçamento sem que haja uma consequência explícita para a imitação, desde que essas crianças tenham uma aquisição da classe geral de comportamentos imitativos, ou seja, já tenham a construção de um repertório de classe de imitação. A isso, Baer e Sherman (1964) denominaram de imitação generalizada.

No presente estudo, partiu-se do pressuposto de que os terapeutas já tinham em seu repertório um comportamento imitativo, qual seja: foi fornecido um modelo e já existindo um repertório estabelecido de imitação, então era de se esperar que ocorresse a imitação generalizada a partir do modelo fornecido pela supervisora. Também é possível afirmar que também não se trata de um procedimento de modelagem, pois tal procedimento envolve a manipulação sistemática das consequências do comportamento, sendo apresentadas de forma diferencial na medida em que o comportamento avança na direção do comportamento final desejado. Então, modelagem é um procedimento no qual não há controle pelo antecedente, mas sim um controle pela consequência apresentada diferencialmente e contingente a aproximações sucessivas ao desempenho final. $\mathrm{O}$ pequeno número de participantes em cada um dos procedimentos adotados dever ser levado em conta quanto a generalidade dos resultados.

\section{DECLARAÇÃO DE CONFLITO DE INTERESSES}

Os autores declaram que não há conflito de interesses relativos à publicação do presente artigo.

\section{CONTRIBUIÇÃO DE CADA AUTOR}

Certificamos que todos os autores participaram suficientemente da escrita desse artigo bem como da condução de todo o trabalho para se tornar pública sua responsabilidade. A contribuição de cada autor pode ser atribuída como se segue: T. L. Duarte, H. J. Guilhardi e R. A. Banaco contribuíram para a concepção do artigo; T. L. Duarte, H. J. Guilhardi e R. A. Banaco foram responsáveis pela formação do desing metodológico; T. L. Duarte fez a coleta de dados; T. L. Duarte e H. J. Guilhardi foram os responsáveis pela redação final.

\section{DIREITOS AUTORAIS}

Este é um artigo aberto e pode ser reproduzido livremente, distribuído, transmitido ou modificado, por qualquer pessoa desde que usado sem fins comerciais. $\mathrm{O}$ trabalho é disponibilizado sob a licença Creative Commons 4.0 BY-NC.

\section{$(\mathrm{oc})$ EY-NC}

\section{REFERÊNCIAS}

Baer, D. M., \& Sherman, J. A. (1964). Reinforcement control of generalized imitation in young children. Journal of Experimental Child Psychology, 1, 37-49. doi: 10.1016/0022-0965(64)90005-0

Baer, D. M, Wolf, M. M., \& Risley, T. R. (1968). Some current dimensions of applied behavior analysis. Journal of Applied Behavior Analysis, 1, 91-97. doi: 10.1901\%2Fjaba.1968.1-91

Banaco, R. A. (1993). O impacto do atendimento sobre a pessoa do terapeuta. Temas em Psicologia, 1(2), 71-79. Ribeirão Preto, SP.

Barker, C., Pistrang, N., \& Elliott, R. (1994). Research methods in clinical and counseling psychology. Chichester: Wiley \& Sons.

Beckert, M. (2002). Relação supervisor-supervisionando e a formação do terapeuta: contribuições da Psicoterapia Analítico Funcional (FAP). Em H. J. Guilhardi (Org.) Sobre Comportamento e Cognição - contribuições para a construção da teoria do comportamento (pp. 245-256). Santo André, SP: ESETEC.

Bitondi, F. R \& Setem, J. (2007). A importância das habilidades terapêuticas e da supervisão clínica: uma revisão de conceitos. Revista Uniara, 20, 203-212. 
Araraquara, SP. doi: $0.25061 / 2527-$ 2675/ReBraM/2007.v11i1.241

Borloti, E. (2004). As relações verbais elementares e o processo autoclítico. Revista Brasileira de Terapia Comportamental e Cognitiva, 6(2), pp. 221-236. São Paulo, SP.

Campos, L. F. L. (1998). Supervisão em terapia cognitivo comportamental. Em B. Rangé (Org.) Psicoterapia Comportamental e Cognitiva - Pesquisa, Prática, Aplicações e Problemas (pp. 357-364). Campinas, SP: Editorial Psy II.

Catania, A. C. (1999). Aprendizagem: comportamento, linguagem e cognição. Trad. D. D. de Souza. Porto Alegre: Artmed Editora.

De Rose, J. C. (1997). O relato verbal segundo a perspectiva da análise do comportamento: contribuições conceituais e experimentais. Em R. A. Banaco (Org.). Sobre comportamento e cognição (Vol. 1, pp. 148-166). São Paulo: Arbytes.

Guilhardi, H. J. (2004). Terapia por Contingências de Reforçamento. Em C. N. Abreu e H. J. Guilhardi (Orgs.) Terapia comportamental e cognitivo comportamental - práticas clínicas $\left(1^{\mathrm{a}} \mathrm{Ed}, \mathrm{pp} .3-40\right)$. São Paulo: Roca.

Matos, M. A. (1997). Com o que o behaviorismo radical trabalha. Em: R. A. Banaco (Org.) Sobre
Comportamento e Cognição (Vol. 1, pp. 56-62). Santo André, SP: ESETEC.

Moreira, S. B. S. (2003). Descrição de algumas variáveis em um procedimento de supervisão de terapia analítica do comportamento. Psicologia: Reflexão e Crítica, 16 (1),157-170. Porto Alegre. doi: 10.1590/S010279722003000100016

Sidman, M. (1960). Tactics of scientific research. New York: Basic Books.

Rosenberg, J. I. (2006). Real-time training: transfer of knowledge through computermediated, real time feedback. Professional Psychology: research and practice, 37 (5), 539-546. doi: 10.1037/07357028.37.5.539

Skinner, B. F. (1953/2003). Por que os organismos se comportam? Ciência e Comportamento Humano, Trad. J. C. Todorov e R. Azzi (11 ${ }^{a}$ Ed., pp. 24-41). São Paulo: Martins Fontes.

Skinner, B. F. (1957/1978). A composição e seus efeitos. Comportamento Verbal, Trad. M. da P. Villalobos (pp. 411-432). São Paulo: Cultrix.

Submetido em: 09/09/2019 Aceito em: 29/12/2019 